\title{
ATOMISTIC MODELING OF CRYSTAL-MELT INTERFACE MOBILITY OF FCC (AL, CU) AND BCC (FE) METALS IN STRONG SUPERHEATING/UNDERCOOLING STATES
}

\author{
V.I. MAZHUKIN*, A.V. SHAPRANOV, O.N. KOROLEVA \\ Keldysh Institute of Applied Mathematics, Russian Academy of Science \\ *Corresponding author. E-mail: vim@ modhef.ru
}

\section{DOI: 10.20948/mathmontis-2020-48-7}

Summary. A detailed study of the mobility and kinetic properties of solid - liquid interfaces (SLI) with different types of crystal lattices (fcc - Al, Cu) and (bcc - Fe) metals in a wide range of temperatures and pressures was carried out using atomistic modeling. The ranges of maximum permissible values of superheated/undercooled states for each metal have been determined. The ultimate goal of the study was to determine the temperature dependences of the stationary front velocity $v_{s \ell}(\Delta T)$ describing the SLI mobility in each of the metals in an analytical form. The analytical dependence $v_{s}(\Delta T)$ was constructed by comparing the results of atomistic modeling in the area of maximum permissible superheating/undercooling values with the data of the main kinetic models of Wilson - Frenkel (WF) and Broughton, Gilmer and Jackson (BGJ). An acceptable agreement was achieved by introducing appropriate correction parameters into the kinetic models using the least squares method. The influence of the crystallographic orientation of metals and external pressure on the SLI mobility is investigated.

\section{INTRODUCTION}

The development and evolution of nanostructures of materials [1] in many cases is determined by the motion of interphase interfaces, and therefore it becomes urgent to determine the main equilibrium and nonequilibrium properties of interfaces.

Equilibrium properties of interfaces are largely related to grain boundaries, which are interfaces between differently oriented crystals of the same material [2]. The importance of studying the structure and thermodynamics of heterophase interfaces of solid materials [3] is determined by the role they play in the stability and morphology of materials. In particular, in situations where the primary solidification process is fully completed, the solid phase, as a rule, can pass into new phases and secondary microstructures [4].

The non-equilibrium properties of mobile solid-liquid interfaces (SLI) are manifested in fast melting and solidification processes, where they play an important role in the establishment of various structural and kinetic properties, the morphology of the growth of a new phase, and non-equilibrium transfer of matter across the phase boundaries [4], [5] .

Initially, the greatest efforts in the atomistic research were devoted to the crystal-melt interfaces in one-component systems. These studies included a detailed analysis of the structure at the interface [6], direct calculations of the interfacial free energies [7], kinetic coefficients, [8,9] and associated crystalline anisotropies [10], as well as other structural and thermodynamic parameters of the solid-liquid interface [11]. At that, the main attention was paid to the determination of the most important property of the mobility of the interphase

2010 Mathematics Subject Classification: 74A20, 74A50, 74A25.

Key words and Phrases: Kinetic models, Kinetic properties, Solid - liquid interface, Superheated/undercooled states, Analytical dependence of front velocity. 
boundary - the speed of heterogeneous phase transformations $v_{s \ell}=v_{s \ell}(\Delta T)$, which depends on the value of superheating/undercooling $\Delta T=T_{s \ell}-T_{m}$ of the interphase boundary $\Gamma(t)$.

According to thermodynamic theory, if the constituent parts of a thermodynamic system are not in equilibrium with each other, then thermodynamic flows arise through their interfaces, accompanied by the process of transformation of matter from one state of aggregation to another one (phase transition). Assuming that the processes occurring in the system are quasi-static, and the fluxes are infinitely small, one can use the methods of equilibrium thermodynamics to describe such a nonequilibrium system. In this case, an infinitesimal difference in the thermodynamic parameters in different parts of the system is assumed. The driving force of first-order phase transitions is the difference in the free energy of two phases at the interface $\Gamma(t)$. Using the theory of thermodynamic potentials, it can be shown that the difference in free energy is linearly proportional to undercooling (superheating) [8, 12]:

$$
\Delta G=\Delta S \cdot \Delta T=\frac{L \Delta T}{T_{e q}}
$$

where $\Delta S, \Delta T$ are the change of the entropy and temperature, $L, T_{e q}$ are the specific heat and equilibrium phase transition temperature.

Identifying the difference in free energy $\Delta G$ as the speed of the phase transformation $v$, one obtains the expression for the speed of the transformation, which in the thermodynamic approach at constant pressure and small deviations from the equilibrium is proportional to superheating/undercooling: $\Delta T: v \approx \mu \Delta T$, where for the case of the solid-liquid interface $\Gamma_{s \ell}(t)$, $\mu$ is the proportionality constant between the normal boundary speed and its superheating/undercooling.

The use of the equilibrium theory of thermodynamic potentials to describe phase transformations (nonequilibrium processes) makes it possible to take into account only a small entry into the metastable superheated/undercooled region and to study phase transformations near the equilibrium line, where the temperature dependence of $v_{s \ell}(\Delta T)$ is mainly controlled by the difference of the free energy of the crystal and liquid phase. This determines the main disadvantages of the thermodynamic approach. Since thermodynamics does not take into account the internal structure of the bodies under consideration, a number of its conclusions and provisions do not have sufficient accuracy and physical clarity.

Structural particles of matter in continuous motion are displayed in the main statements of the molecular kinetic theory, in which all processes are considered at the atomic or molecular level, and the particles have a Maxwellian velocity distribution.

The simplest kinetic dependence in the form of the linear growth rate of a crystal from a melt, which outwardly agrees well with the above thermodynamic relationship, was obtained on the basis of classical molecular kinetic models. In this dependence, the proportionality constant is called the kinetic coefficient $[14,15]$. The main application of the kinetic coefficient and its various modifications $[16,17]$ was found in the description of melting solidification processes in the vicinity of the equilibrium melting temperature $T_{s} \approx T_{m}$, where the coefficient $\mu$ is the main parameter characterizing the mobility of the SLI. In the kinetic approach in the most general form, the coefficient $\mu$ is written as $[9,18]$ :

$$
\mathrm{U}_{\langle h k \ell\rangle}(\Delta \mathrm{T})=\mu_{\langle h k \ell\rangle} \Delta T
$$


where $\langle h k \ell\rangle$ are the indices characterizing the anisotropy of the kinetic coefficient and indicating the dependence of the SLI rate on the crystallographic orientation of the interface. The anisotropy of the kinetic coefficient and its value are the most important factors affecting the growth rate and the associated morphology of solidification at small undercooling.

The need to consider an extended range of superheated/undercooled states was caused by a large number of theoretical [19 - 24] and experimental [25-27] works that showed that the kinetics of melting/solidification processes far from the equilibrium melting temperature $T_{m}$ is significantly different from the kinetics in the vicinity of $T_{m}$.

In this work, using atomistic modeling, a detailed study of the mobility and kinetic properties of SLI with different lattices (fcc - Al, Cu) and (bcc - Fe) and crystallographic orientations for metals is carried out in a wide range of temperatures and pressures. The ultimate goal of the study is to construct the temperature dependences of the stationary front velocity describing the SLI mobility in metals in a wide range of maximum permissible values of superheating/undercooling. The analytical dependences of $v_{s \ell}(\Delta T)$ were determined by comparing the results of the atomistic modeling in the area of maximum permissible values of superheating/undercooling with the data of the kinetic models. An acceptable match is achieved by introducing the corresponding correction parameters into the model [28], [29].

\section{MAIN KINETIC MODELS}

Traditionally, the kinetic theories have developed most intensively in the direction of the processes of crystallization - solidification of melts, which is explained by the large content in them (along with the fundamental aspects) of a large number of technological applications, in which crystallization of metastable phases in deeply supercooled melts leads to significant structural changes associated with thermodynamic, kinetic and mechanical properties of materials.

Kinetic theories depicting temperature dependences $v_{s \ell}(\Delta T)$ of SLI mobility are based on various physical phenomena. The most well-known kinetic theories include the classical Wilson - Frenkel (WF) theory [30-32] with a diffusion mechanism for controlling the interface kinetics, the kinetic model of Broughton, Gilmer and Jackson (BGJ) [33] with a collisional thermal mechanism, and the kinetic theory [7,34], with the mechanism of influence of density changes, the so-called density functional theory (DFT).

In the kinetic theory of WF, [30-32], the interface speed is related to the diffusion of atoms in the liquid phase. This theory is often called the transition state theory, since it is based on the assumption that melting or solidification occurs through some intermediate or transition state. In this theory, the SLI rate is controlled by a diffusion limiting mechanism. This mechanism is based on the assumption that atoms (molecules) must overcome the diffusion barrier during the transition from liquid to solid phase. The transition is accompanied by a significant restructuring of the interface. In this case, the rate of the crystallization process is assumed to be proportional to the diffusion coefficient, which is usually presented in the form of the Arrhenius equation

$$
D=D_{0} \exp \left(-\frac{Q}{k_{B} T_{s \ell}}\right)
$$


where $Q$ is the energy of activation for diffusive motion in liquid, $k_{B}$ is the Boltzmann constant, $k_{B} T$ is the average thermal energy for a single atom, $D_{0}$ is the prefactor determining the speed of the process.

Temperature dependence of the crystallization / melting front velocity $v_{s \ell}(\Delta T)$ in the model with diffusive limitation is expressed in the generalized form as:

$$
\left[\mathrm{v}_{s \ell}(\Delta T)\right]_{\langle h k \ell\rangle}^{\mathrm{WF}}=\frac{a f}{\lambda^{2}} D\left[\exp \left(\frac{\Delta G}{k_{B} T}\right)-1\right]=C_{\langle h k \ell\rangle}^{\mathrm{WF}} \frac{D}{a}\left[\exp \left(\frac{L_{m}}{k_{B} T_{m}} \frac{\Delta T}{T_{s \ell}}\right)-1\right]
$$

where $C_{\langle h k \ell\rangle}^{\mathrm{WF}}=\frac{a^{2}}{\lambda^{2}} f, a$ is the interatomic distance, $\lambda$ is the man free path for the atoms of this process, it is assumed to be proportional to the lattice constant, $a: \lambda<a, f$ is the efficiency coefficient (a constant of the order of unity, $f<1$ ), characterizing the fraction of collisions of liquid atoms with solid, which leads to crystallization. These quantities do not have a rigorous definition, are difficult to measure, and, moreover, depend on the crystallographic orientation of the interface $[18,35]$.

The BGJ theory [33], originally proposed as an improvement on the earlier WF theory, uses the frequency of thermal collisions of atoms with the interface [36] as a constraint. The modification of the WF transition state theory was motivated by the results of MD simulation [33] performed with the Lennard-Jones potential, which was assumed to be metal-like, which showed that the growth of crystals of monatomic systems may not in all cases be limited by diffusion. In particular, in the region of very low temperatures, the diffusion coefficient tends to zero, but according to the simulation results, the SLI rate is still finite and the WF model turns out to be wrong. Following the hypothesis [36] that the solidification of monatomic metals is limited only by the frequency of collisions of melt atoms with the crystal surface, the authors of the BGJ model [33] replaced the diffusion term in (3) with the average thermal velocity of atoms $v_{T}=\sqrt{3 k_{B} T_{s \ell} / m}$

$$
\left[\mathrm{U}_{s \ell}(\Delta T)\right]_{\langle h k \ell\rangle}^{\mathrm{BGJ}}=\frac{a}{\lambda} f_{0} v_{T}\left[\exp \left(\frac{L_{m}}{k_{B} T_{m}} \frac{\Delta T}{T_{s \ell}}\right)-1\right]=C_{\langle h k \ell\rangle}^{\mathrm{BGJ}} \sqrt{\frac{3 k_{B} T_{s \ell}}{m}}\left[\exp \left(\frac{L_{m}}{k_{B} T_{m}} \frac{\Delta T}{T_{s \ell}}\right)-1\right]
$$

where $C_{(h k \ell)}^{B G J}=\frac{a}{\lambda} f_{0}$ is a dimensionless coefficient, $m$ is the atom mass.

In the kinetic theory developed on the basis of DFT [7, 34], the interface kinetics is controlled by the relaxation of short-wave density waves. Density functional theory also explicitly explains the anisotropy of the coefficient $\mu_{\{h k \ell}$, confirming that it is determined only by $\langle h k \ell\rangle$ factors.

Within the framework of the kinetic approach it is easy to formulate an analytical form of the kinetic coefficient $\mu_{\{h k \ell}(1)$. For the temperatures in the vicinity of the equilibrium melting temperature $T_{m},\left(T_{s \ell} \approx T_{m}\right)$ from the Eqs. (3) и (4) one can obtain the coefficient $\mu_{\{h k \ell}$ in the form:

$$
\mu_{\langle h k \ell\rangle}^{\mathrm{WF}}=C_{\langle h k \ell\rangle}^{\mathrm{WF}} \frac{D}{a} \frac{L_{m}}{k_{B} T_{m}^{2}}
$$




$$
\mu_{\langle h k \ell\rangle}^{B G J}=C_{\langle h k \ell\rangle}^{\mathrm{BGJ}} \sqrt{\frac{3 k_{B} T_{m}}{m}} \frac{L_{m}}{k_{B} T_{m}^{2}}
$$

It is easy to see that the equations (5), (6) represent the first term in the expansion of equations (3), (4) in the neighborhood of $T_{m}$. The dimensionless coefficients $C_{\langle h k \ell\rangle}^{\mathrm{WF}}, C_{\langle h k \ell\rangle}^{B G J}$ do not have a strict definition and depend on the crystallographic orientation of the interface [6].

The presence of the values without strict definition $Q, D_{0}, \lambda$ and dimensionless coefficients $\left.f, C_{\langle h k \ell\rangle}^{\mathrm{WF}}, C_{\langle h k \ell\rangle}^{B G J}\right)$ the models (3) - (6) required further investigation of the kinetics of the phase transformations. It was performed using atomistic modeling. Modeling based on molecular dynamic methods using both pair Lennard-Jones [37] and many-particles EAM [4, 5, 23,35,38 - 41] potentials for metallic interfaces with different crystallographic orientation $\langle 100\rangle,\langle 110\rangle$ and $\langle 111\rangle$ allowed to find that both theories (models WF (3) и BGJ (4)) near the equilibrium temperature $T_{m}$ give the results that are in reasonable agreement between themselves and the results of theory (DFT) [7, 34].

With the development of more accurate many-particle potentials for metals, a number of molecular dynamics studies of crystal growth in pure metals were carried out. Atomistic modeling [23, 27] showed that in the range of values close to the melting point, the crystallization process can be represented with acceptable accuracy by kinetic models with diffusion (3) and collisional thermal constraints (4), as well as by models of the density functional theory [7, 34]. For deep undercooling, it is preferable [23, 27] to use the kinetic model with collisional thermal constraint [33] and the Arrhenius-type model [35]. In the region of intermediate undercooling at a level of $\sim 0.7 T_{m}$, the advantage is retained by the model of the transition state with diffusion limitation of WF. In the paper [42], a specially developed semi-empirical potential was used to simulate the phase transformation in a disordered one-component system. The modeling showed that the WF theory satisfactorily describes the results of MD simulation of interface migration in the temperature range from $0.55 T_{m}$ to $T_{m}$, while the BGJ theory is less accurate in describing the temperature dependence of the SLI speed in the same temperature range. Below $0.55 T_{m}$, none of the existing theories is able to reproduce the temperature dependence of the interface speed.

Recent use of ultrashort fs, ps - laser pulses to achieve deep undercooling in melts of thin (10-50 nm) metal films [23-27] has brought to the fore the study of bulk and surface mechanisms of melting of solids $[43,44]$. The degree of undercooling during solidification can be easily controlled by varying the thickness of the thin films. The problem of determining the mobility of the crystal-melt interface during melting / solidification of metals [49] as a function of temperature $v_{s \ell}(\Delta T)$ in the entire range of deep superheating/undercooling became urgent especially with the subsequent transition to the problems of crystal growth from deeply undercooled melts [45, 46], as well as kinetic glass transition of undercooled liquids [47, 48].

Since in the overwhelming majority of works $[5,9-11,18,23,27,35,42]$ the study of the temperature dependence of the stationary speed $v\left(T_{s}\right)$ was carried out in the temperature range of crystallization with deep undercooling, the important question of the possibility of using the analyzed kinetic models remained open: is it possible to determine the speed of movement of a SLI with acceptable agreement in the temperature range of melting with strong superheating of the solid phase? In the literature there is a small number of works [28, 
29, 49-54], in which the kinetic models (3), (4) were tested in a wide temperature range, covering the processes of crystallization and melting.

\section{STATEMENT OF THE PROBLEM AND COMPUTATIONAL ALGORITHM}

The determination of the stationary temperature dependence of the kinetic rate of SLI in the range of maximum permissible values of superheating/undercooling was carried out using a computational experiment consisting of a large series of molecular dynamics calculations.

Metals of two types were involved in the modeling: with fcc lattice - aluminum (Al), copper $(\mathrm{Cu})$; and bcc lattice - iron $(\mathrm{Fe})$ with different crystallographic orientations. Atomistic models are based on the model concept of a polyatomic molecular system in which all atoms are represented by material points, the motion of which is described in the classical case by Newton's equations. As a result, the evolution of an ensemble of $N$ point particles is described by a system of $2 N$ ordinary differential equations. The interaction between particles was described by various many-particle EAM potentials: for aluminum [38], copper [39], and for iron [40] with parameterization [41]. The integration of this system of equations for all $N$ particles requires the knowledge of the coordinates and velocities $\left.\left(\vec{r}_{i}, \vec{v}_{i}\right)\right|_{t=0}$ at the initial time $t=0$. For $\mathrm{Al}$ (and in a similar way for other metals), the computational domain was set in the form of a parallelepiped with a size of $5 \times 5 \times 41 \mathrm{~nm}$ and filled with 57600 particles. All the atoms of the parallelepiped were formed in the form of a set of $20 \times 10 \times 10$ lattice unit cells. The periodic boundary conditions were set in all three spatial directions at the boundaries of the computational domain, i.e. the simulated object was an infinite single crystal of metal.

The initial state of the computational domain for modeling of the process of heterogeneous melting of a metal is a solid-state structure with a liquid layer in the middle of the computational domain, in which the crystalline and liquid phases are separated by two flat interfaces. To study the melting process, the liquid phase occupies about $18 \%$ of the volume of the computational domain, and to study the crystallization process $\sim 80 \%$. Subsequently, the interface speed was measured directly as a function of its temperature.

In the course of calculations using a thermostat, a fixed temperature value was established and maintained throughout the entire computational domain during the entire numerical experiment. At the same time, the barostat kept a constant value of the external pressure. This excluded the reverse effect of the release / absorption of the latent heat of fusion on the local temperature at the fronts. As a result, the process of heterogeneous melting / crystallization quickly reaches a stationary regime, and the change in the amount of a new phase occurs almost linearly. The position of the melting-crystallization fronts was tracked using the order parameter.

The maximum permissible values of overheating/overcooling are understood as the temperatures at which, in the event of overheating, the initial crystal still retains mechanical stability, the loss of which is associated with the onset of homogeneous melting. For stationary action conditions, the value of the limiting superheating is $T_{s} \ell \approx 1.25 T_{m}$ which is in good agreement with the results of [43]. Under unsteady action, the limiting value of overheating reached the value of $1.5 T_{m}$ or more, which coincides with the estimates [55].

In the case of undercooling, the limiting temperature is the temperature at which the undercooled melt is still pure liquid. The limitation of deep penetration into the metastable undercooled region is associated with the formation of an intermediate (interstitial) phase, for 
which the order parameter turns out to be much larger than that of a liquid, but much less than that of a normal crystal. The appearance of the interstitial phase indicates the beginning of the vitrification process. The glass transition temperature for most metals is in the region $T_{c} \approx(0.3 \div 0.5) T_{m}$ [35]. The variants of calculations in which a noticeable proportion of the interstitial phase appeared were excluded from consideration.

Taking into account the above estimates in the calculations, the temperature range of the superheating/undercooling limit values was chosen within $T_{s \ell} \approx(0.3 \div 1.25) T_{m}$. For the considered metals $\mathrm{Cu}$, and $\mathrm{Fe}$, the influence of the crystallographic orientation of the interface in the plane $\langle 100\rangle$ was considered and for $\mathrm{Al}-\langle 100\rangle,\langle 111\rangle$. The influence of external pressure for two values, 0 and $80 \mathrm{kbar}$, was also considered for each of the metals.

\section{THE RESULTS OF THE ATOMISTIC MODELING. THE CONSTRUCTION OF AN ANALYTICAL DEPENDENCE $v_{\langle h k \ell\rangle}(\Delta T)$}

The result of the molecular dynamics simulation was obtaining a discrete set of values of the phase front velocity depending on the crystallographic orientation of the interface: for $\mathrm{Al}$ : $v_{\{100\rangle}(\Delta T)$, for $\mathrm{Cu}$ and $\mathrm{Fe}: v_{\{100\rangle}(\Delta T)$ in the range of extreme values of superheating/undercooling. Discrete values $v_{\{h k \ell}(\Delta T)$ in Fig. 1, 2, 3, 4 are marked with markers (circles and triangles $-P=80 \mathrm{kbar}$ ).

The analytical dependencies were constructed using the least squares method.

The discrete set of values $v_{\{h k \ell}\left(T_{s \ell}\right)$ obtained from the molecular dynamics modeling was compared with the results of the kinetic models of BGJ [33], (equation (4)) and WF [30-32], (equation (3)). When comparing, it was taken into account that equations (3), (4) contain two thermophysical parameters - the equilibrium melting temperature $T_{m}$ and the latent heat of melting $L_{m}$, the values of which, due to the peculiarities of the interaction potentials used in molecular dynamics calculations, may slightly differ from the reference ones. To correctly compare the MD data with the kinetic data in equations (3), (4), we used the values of $T_{m}$ and $L_{m}$ determined for all considered metals $\mathrm{Al}, \mathrm{Cu}, \mathrm{Fe}$ from additional $\mathrm{MD}$ calculations performed by the method [56] with the same potentials [38], [ 39], [40, 41]. The calculation results for 2 values of external pressure are shown in Table 1.

\begin{tabular}{|c|c|c|c|}
\hline \multirow{2}{*}{ Metal } & Pressure $[\mathrm{kbar}]$ & $T_{m},[\mathrm{~K}]$ & $L_{m},[\mathrm{~kJ} / \mathrm{mol}]$ \\
\hline \multirow{2}{*}{$A l$} & 0 & 949 & 8.90 \\
\cline { 2 - 4 } & 80 & 1332 & 11.30 \\
\hline \multirow{2}{*}{$C u$} & 0 & 1315 & 11.48 \\
\cline { 2 - 4 } & 80 & 1602 & 13.21 \\
\hline \multirow{2}{*}{$F e$} & 0 & 1775 & 15.57 \\
\cline { 2 - 4 } & 80 & 2062 & 17.14 \\
\hline
\end{tabular}

Table 1. The calculation results of $T_{m}$ and $L_{m}$ for 2 values of external pressure 
The model of BGJ. Full alignment of a discrete set of values $v_{\{h k \ell}(\Delta T)$ with equation (4) was achieved using a 2-parametric $C_{\{h k \ell \ell}, \beta_{\{h k \ell\rangle}$ approximation:

$$
\left[\mathrm{v}_{s \ell}(\Delta T)\right]_{\langle h k \ell\rangle}^{\mathrm{BGJ}}=C_{\langle h k \ell\rangle}^{B G J} \sqrt{\frac{3 k_{B}}{m} T_{s \ell}}\left[\exp \left(\beta_{\langle h k \ell\rangle}^{B G J} \frac{L_{m}(P)}{k_{B} T_{m}(P)} \cdot \frac{T_{s \ell}-T_{m}(P)}{T_{s \ell}}\right)-1\right]
$$

where $C_{\langle h k \ell\rangle}^{B G J}, \beta_{\langle h k \ell\rangle}^{B G J}$ are the approximation parameters. By introducing additional parameters $C_{\langle h k \ell\rangle}^{B G J}$ before the whole expression and $\beta_{\langle h k \ell\rangle}^{B G J}$ in the exponent, it is possible to achieve the required precision of the temperature dependence $v_{\langle h k \ell\rangle}^{\mathrm{BGJ}}(\Delta T)$ in a wide temperature range. The values of the parameters $C_{\langle h k \ell\rangle}^{B G J}$ found from MD calculations allow to automatically estimate the value of $\frac{a}{\lambda} f$, which is not very precisely determined in the BGJ model.

\begin{tabular}{|c|c|c|c|c|}
\hline Metal & $\begin{array}{c}\text { Pressure } \\
\text { [kbar] }\end{array}$ & $C_{\langle 100\rangle}^{B G J}$ & $\beta_{\langle 100\rangle}^{\mathrm{BGJ}}$ & $\begin{array}{c}\sigma \\
{[\mathrm{m} / \mathrm{s}]}\end{array}$ \\
\hline \multirow{2}{*}{$\begin{array}{c}\mathrm{Al} \\
\mathrm{\cup}_{\langle 100\rangle}^{\mathrm{BGJ}}(\Delta T)\end{array}$} & $\mathrm{P}=0$ & 0.344 & 5.01 & 5.97 \\
\hline & $\mathrm{P}=80$ & 0.374 & 5.37 & 8.00 \\
\hline \multirow{2}{*}{$\begin{array}{c}\mathrm{Cu} \\
\mathrm{v}_{\langle 100\rangle}^{\mathrm{BGJ}}(\Delta T)\end{array}$} & $\mathrm{P}=0$ & 0.416 & 5.74 & 11.75 \\
\hline & $\mathrm{P}=80$ & 0.441 & 5.51 & 12.36 \\
\hline \multirow{2}{*}{$\begin{array}{c}\mathrm{Fe} \\
\mathrm{v}_{\langle 100\rangle}^{\mathrm{BGJ}}(\Delta T)\end{array}$} & $\mathrm{P}=0$ & 0.365 & 6.34 & 3.89 \\
\hline & $\mathrm{P}=80$ & 0.416 & 6.31 & 8.56 \\
\hline \multirow{2}{*}{$\begin{array}{c}\mathrm{Al} \\
\mathrm{U}_{\langle 111\rangle}^{\mathrm{BGJ}}(\Delta T)\end{array}$} & \multirow{2}{*}{$\mathrm{P}=0$} & $C_{\langle 111\rangle}^{\mathrm{BGJ}}$ & $\beta_{\langle 111\rangle}^{\mathrm{BGJ}}$ & $\begin{array}{c}\sigma \\
{[\mathrm{m} / \mathrm{s}]}\end{array}$ \\
\hline & & 0.165 & 7.27 & 5.11 \\
\hline
\end{tabular}

Table 2. The values of the approximation parameters and the mean square deviation $\sigma$ of the approximating function $\mathrm{v}_{\langle h k \ell\rangle}^{\mathrm{BGJ}}(\Delta T)$ from the discrete set of values $\mathrm{v}_{\langle h k \ell\rangle}(\Delta T)$.

The best agreement, with an error not exceeding a few percent, over the entire temperature range was achieved with the values of the approximating parameters $C_{\langle h k \ell\rangle}^{B G J}, \beta_{\langle h k \ell\rangle}^{B G J}$ presented in Table 2.

In Figures 1, 2, 3, solid red and dashed blue lines show the plotted dependences of the SLI speed in the crystallographic planes $\langle 100\rangle$ and $\langle 111\rangle$ at the external pressure $P=0$ and $P=80$ kbar for the elements $\mathrm{Al}, \mathrm{Cu}, \mathrm{Fe}$.

In Fig. 4 for $\mathrm{Al}$, the solid red and dashed black lines show the plotted stationary dependences of the SLI speed in the crystallographic planes $\langle 100\rangle$ and $\langle 111\rangle$ and at external pressure $P=0$. 


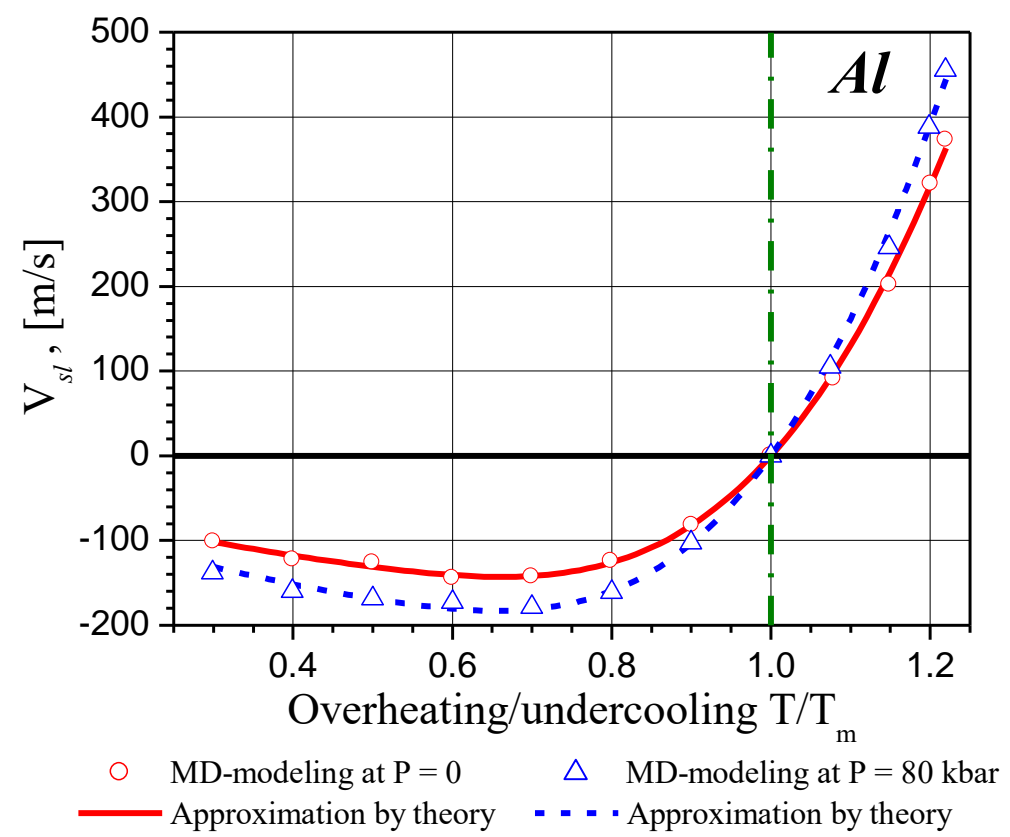

Fig. 1. The dependence of the SLI rate in the crystallographic plane $\langle 100\rangle$ on the superheating/undercooling value for $\mathrm{Al}$ at $P=0$ and $P=80 \mathrm{kbar}$.

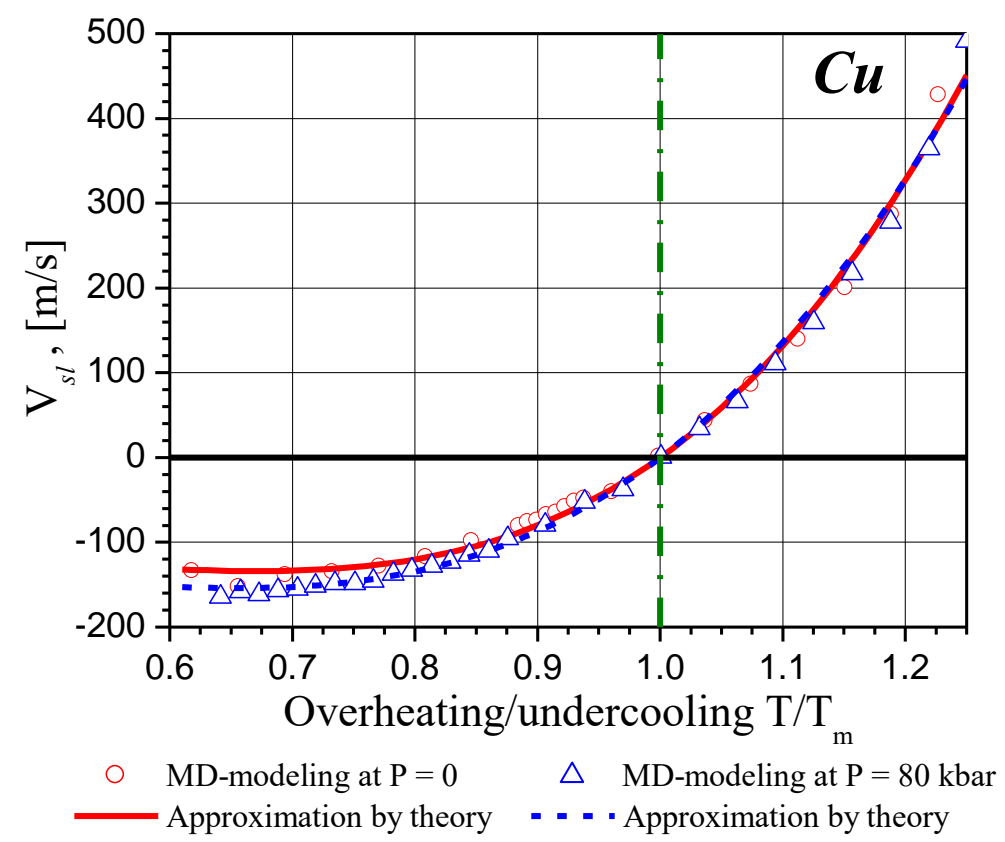

Fig. 2. The dependence of the SLI rate in the crystallographic plane $\langle 100\rangle$ on the superheating/undercooling value for $\mathrm{Cu}$ at $P=0$ and $P=80 \mathrm{kbar}$. 


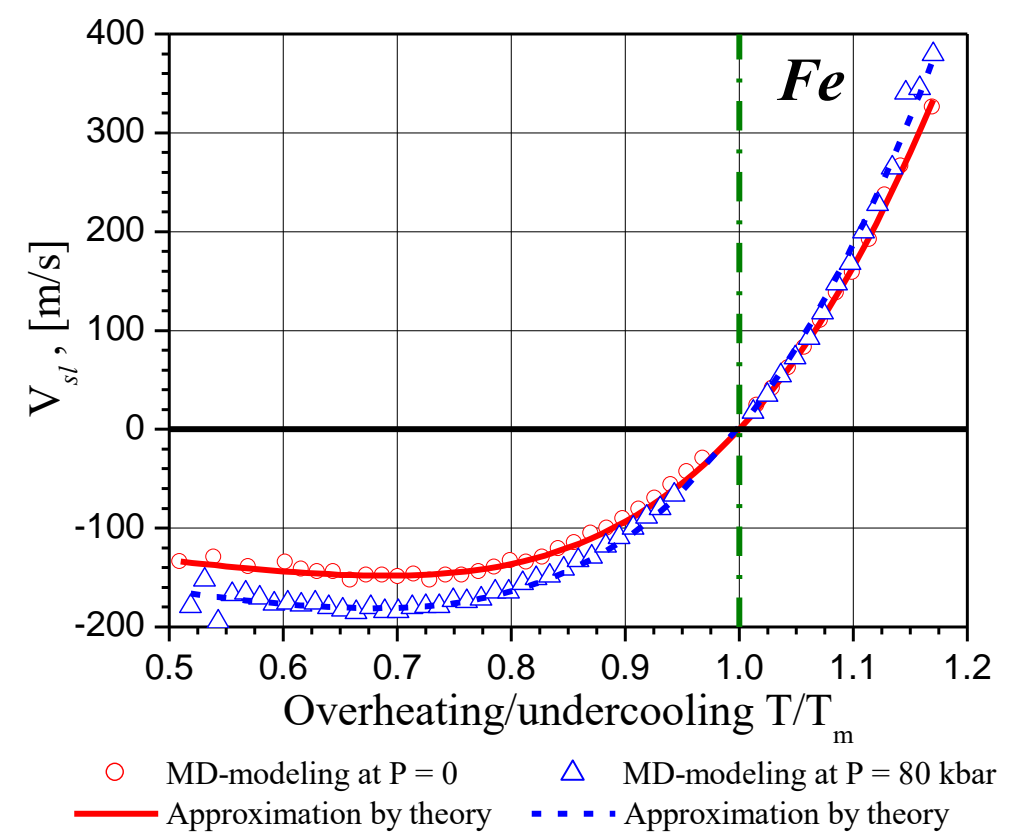

Fig.3 The dependence of the SLI rate in the crystallographic plane $\langle 100\rangle$ on the superheating/undercooling value for Fe at $P=0$ and $P=80$ kbar.

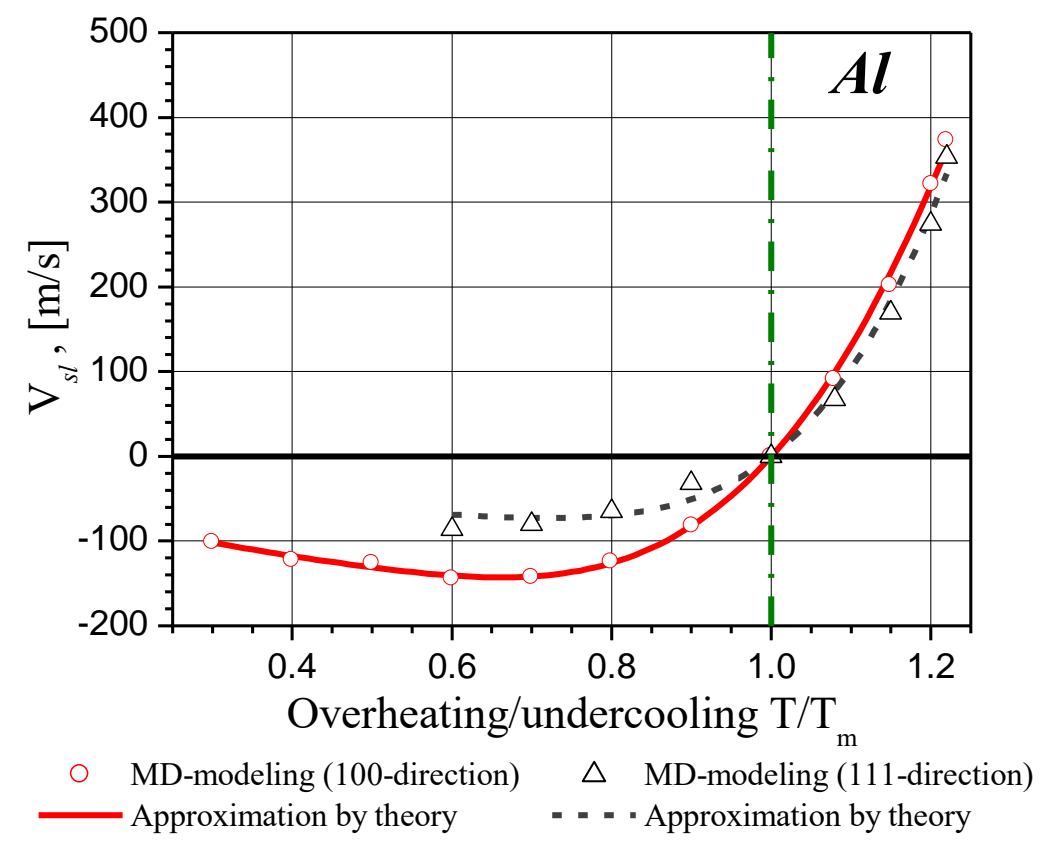

Fig. 4. The dependence of the SLI rate in the crystallographic planes $\langle 100\rangle$ and $\langle 111\rangle$ on the superheating/undercooling value for $\mathrm{Al}$ at $P=0$. 
Note that when simulating the crystallization of aluminum in the region of significant undercooling of the interface with the orientation $\langle 111\rangle$, the probability of the appearance of stacking faults significantly increases. Their accumulation leads to a curvature of the initial crystallographic plane and the further propagation of the front is going in a direction different from $\langle 111\rangle$. For this reason in the crystallographic direction $\langle 111\rangle$, it was not possible to enter the undercooling region below $0.6 \times T_{m}$.

The model of WF. The diffusion limited model [30 - 32] was also used to approximate the discrete set of values $v_{\{h k \ell}\left(T_{s \ell}\right)$ from molecular dynamics modeling for aluminum at two values of external pressure $(P=0, P=80 \mathrm{kbar})$.

A complete fit of the discrete values $v_{\{h k \ell\rangle}(\Delta T)$ to the equation (5) was reached using a three-parameter $C_{\langle h k \ell\rangle}^{\mathrm{WF}}, \beta_{\langle h k \ell\rangle}^{W F}, Q_{\langle h k \ell\rangle}$ approximation:

$$
\left[\mathrm{v}_{s \ell}(\Delta T)\right]_{\langle h k \ell\rangle}^{W F}=C_{\langle h k \ell\rangle}^{W F} \operatorname{dim}\left[\frac{D_{0}}{a}\right] \exp \left(-\frac{Q_{\langle h k \ell\rangle}}{k_{B} T_{s \ell}}\right)\left[\beta_{\langle h k \ell\rangle}^{W F} \exp \left(\frac{L_{m}(P)}{k_{B} T_{m}(P)} \frac{T_{s \ell}-T_{m}(P)}{T_{s \ell}}\right)-1\right]
$$

In the considered range of the limiting values of superheating/undercooling in the crystallographic plane $\langle 100\rangle$, the best fit with an error less than few percent was reached with the values of the approximation parameters $C_{\langle 100\rangle}^{\mathrm{WF}}, \beta_{\langle 100\rangle}^{\mathrm{WF}}, Q_{\langle 100\rangle}$ listed in the Table 3. The value of $C_{\langle 100\rangle}^{\mathrm{WF}}$ was determined keeping in mind that $\operatorname{dim}\left[\frac{D_{0}}{a}\right]=(\mathrm{m} / \mathrm{s})$ is only a dimensionality of the relation $D_{0} / a$.

\begin{tabular}{|c|c|c|c|c|c|}
\hline Metal & $\begin{array}{c}\text { Pressure } \\
\text { [kbar] }\end{array}$ & $\begin{array}{c}C_{\langle 100\rangle}^{\mathrm{WF}} \\
{[\mathrm{m} / \mathrm{s}]}\end{array}$ & $\begin{array}{c}Q_{\langle 100\rangle} \\
{[\mathrm{kJ} / \mathrm{mol}]}\end{array}$ & $\beta_{\langle 100\rangle}^{\mathrm{WF}}$ & $\begin{array}{c}\sigma \\
{[\mathrm{m} / \mathrm{s}]}\end{array}$ \\
\hline \multirow{2}{*}{$\begin{array}{c}\mathrm{Al} \\
\mathrm{U}_{\langle 100\rangle}^{\mathrm{WF}}(\Delta T)\end{array}$} & $\mathrm{P}=0$ & 198.9 & 1.58 & 5.65 & 5.82 \\
\hline & $\mathrm{P}=80$ & 233.8 & 1.73 & 6.28 & 6.90 \\
\hline \multirow{2}{*}{$\begin{array}{c}\mathrm{Cu} \\
\mathrm{v}_{\langle 100\rangle}^{\mathrm{WF}}(\Delta T)\end{array}$} & $\mathrm{P}=0$ & 140.64 & 0 & 6.88 & 10.70 \\
\hline & $\mathrm{P}=80$ & 163.18 & 0 & 6.67 & 11.48 \\
\hline \multirow{2}{*}{$\begin{array}{c}\mathrm{Fe} \\
\mathrm{U}_{\langle 100\rangle}^{\mathrm{WF}}(\Delta T)\end{array}$} & $\mathrm{P}=0$ & 251.02 & 0.051 & 6.63 & 3.87 \\
\hline & $\mathrm{P}=80$ & 244.11 & 0.033 & 7.10 & 8.31 \\
\hline
\end{tabular}

Table 3. The values of the approximation parameters and the mean square deviation $\sigma$ of the approximating function $\mathrm{v}_{\langle 100\rangle}^{\mathrm{WF}}(\Delta T)$.

A comparison of $v_{\langle h k \ell}^{\mathrm{WF}}(\Delta T)$ (8) with the curves $v_{\langle h k \ell}^{\mathrm{BGJ}}(\Delta T)$, (7), at Figs. $(1-3)$ showed almost complete fit with an error less than $1 \%$. For this reason in this paper, a separate plot of $\mathrm{v}_{\langle h k \ell\rangle}^{\mathrm{WF}}(T)$ is not provided. 


\section{SHORT ANALYSIS}

The obtained curves $\mathrm{v}_{\langle h k \ell\rangle}^{\mathrm{WF}, \mathrm{BGJ}}(\Delta T)$, characterizing the mobility of SLI, for the considered (fcc) and (bcc) metals in the crystallographic plane $\langle 100\rangle$ have a great generality. The melting branches in the range $T_{m} \leq T_{s \ell} \leq 1.25 T_{m}$ have exponential behavior as the superheating rises, reaching the maximum values $v_{s \ell}^{W F, B G J}(\Delta T) \sim 300 \div 350 \mathrm{~m} / \mathrm{s}$. The crystallization process in the undercooling region $0.5 T_{m}<T_{s} \leq T_{m}$ takes place in a more complicated manner. The speed of crystallization $\cup_{s \ell}^{W F, B G J}(\Delta T)$ at all curves, Figs.1-3, in the vicinity of $T_{s \ell} \sim 0.7 T_{m}$ have a wellnoticeable maximum of $\sim 140 \div 160 \mathrm{~m} / \mathrm{s}$. It should be noted that the maximum crystallization rate for Fe coincides with similar data obtained in [35]. The appearance of the maximum in the crystallization rate is associated with the beginning of the formation of the interstitial phase, which slows down the speed of the phase front. In this work, only crystallization processes in a undercooled pure liquid are considered. Glass transition processes occurring near and below the temperature $T_{g}, 0<T_{s \ell} \leq T_{g}$, are not included in the consideration, since the complexity and importance of this process, in particular, for technological applications deserves a separate consideration.

External pressure $(P=80 \mathrm{kbar})$ has no significant effect on the behavior of the kinetic speed in the entire considered range of superheating/undercooling (blue curves). But the maximum values of the rate of melting and crystallization in this case increase to $350 \div 450$ $\mathrm{m} / \mathrm{s}$ and $160 \div 180 \mathrm{~m} / \mathrm{s}$, respectively. To a much greater extent, SLI mobility is affected by its crystallographic orientation. For example, for aluminum, the ratio of the maximum crystallization rates reaches $v_{\langle 100\rangle}^{\mathrm{BGJ}}(\Delta T) / \mathrm{v}_{\langle 111\rangle}^{\mathrm{BGJ}}(\Delta T) \approx 2$ times, and that of melting, 1.2 times, Fig. 4.

\section{CONCLUSIONS}

The molecular dynamics method was used to study the kinetics of melting/crystallization of (fcc) and (bcc) metals ( $\mathrm{Al}, \mathrm{Cu}, \mathrm{Fe}$ ) in the range of limiting values of superheating/undercooling. The limiting superheating/undercooling for each of the metals is determined by the temperature values at which the superheated crystal retains the properties of the crystal, and the undercooled melt still remains liquid.

a) The modeling results showed that for the considered metals, the range of the limiting values of superheating/undercooling is in the range $T_{s l} \approx(0.3 \div 1.25) T_{m}$ for $\mathrm{Al}_{\langle 100\rangle}$, $T_{s \ell} \approx(0.6 \div 1.2) T_{m}$ for $\mathrm{Al}_{\langle 111\rangle}, T_{s \ell} \approx(0.6 \div 1.3) T_{m}$ for $\mathrm{Cu}, T_{s \ell} \approx(0.65 \div 1.18) T_{m}$ for Fe.

b) A discrete set of velocity values $v_{\{h k \ell}\left(T_{s \ell}, P\right)$ obtained from the atomistic modeling together with the kinetic models of WF (3) and BGJ (4) were used to construct analytical dependences of the stationary velocity of motion of the SLI $v_{\langle h k \ell\rangle}^{W F, B G J}(\Delta T, P)$ (Eqs. $(7,8)$ ) over the entire range of limiting values of superheating/undercooling.

c) In the considered range of limiting superheating/undercooling, both kinetic models of WF and BGJ allow, with practically the same error of $\sim 1 \%$, to construct analytical expressions for the velocities in the form of two- and three-parametric curves in the case of using models of BGJ (4) and WF (3) respectively. 
d) The temperature dependences of the solid/liquid interface velocity for $\mathrm{Al}, \mathrm{Cu}$, and $\mathrm{Fe}$, determined from the results of simulations using different crystallographic planes, demonstrate a clear asymmetry with respect to the melting point $T_{m}$, which is explained by the strong difference between the melting kinetics in a highly superheated state and the kinetics of solidification in a highly undercooled state.

e) For all metals, the change in the temperature dependence of the velocity $v_{s t}(\Delta T)$ when passing through the melting point $T_{m}$ occurs smoothly without a bend in the slope.

f) The crystallographic orientation of the metal, rather than its crystal lattice type, has the greatest impact on SLI mobility.

g) External pressure has no significant effect on the kinetic velocity behavior over the entire superheating/undercooling range under consideration, but leads to an increase in the maximum melting and crystallization rates.

h) The obtained results of atomistic modeling indicate that the capabilities of the classical Wilson - Frenkel model [30-32] were greatly underestimated.

Acknowledgements: This study was supported by the Russian Science Foundation (project no. 18-11-00318).

\section{REFERENCES}

1. Handbook of Materials Modeling, Vol. 1, 2, Yip Sidney (Ed.), Springer, Dordrecht, Berlin, Heidelberg, New York (2005).

2. K. Thornton, J. Ågren, P.W. Voorhees, "Modelling the evolution of phase boundaries in solids at the meso- and nano-scales", Acta Materialia, 51 (19), 5675-5710 (2003).

3. A.P. Sutton, R.W. Balluffi, Interfaces in crystalline materials, Oxford: Clarendon Press (1995).

4. Y. Mishin, M. Asta, Ju Li. "Atomistic modeling of interfaces and their impact on microstructure and properties. Overview № 148”, Acta Materialia, 58(4), 1117-1151, (2010). https://doi.org/10.1016/j.actamat.2009.10.049

5. J.J. Hoyt, M. Asta, A. Karma, "Atomistic and continuum modeling of dendritic solidification", Materials Science and Engineering R, 41, 121-163 (2003).

6. M. Asta, C. Beckermann, A. Karma, W. Kurz, R. Napolitano, M. Plappf, G. Purdy, M. Rappaz, R. Trivedi, "Solidification microstructures and solid-state parallels: Recent developments, future directions. Overview No. 146", Acta Materialia, 57, 941-971 (2009).

7. Yu C. Shen and David W. Oxtoby, "Density functional theory of crystal growth: Lennard-Jones fluids", J. Chem. Phys., 104 (11), 4233 - 4242 (1996). doi: 10.1063/1.471234.

8. G.H. Rodway, J.D. Hunt, "Thermoelectric investigation of solidification of lead. I. Pure lead", $J$. Cryst. Growth, 112 (2-3), 554-562 (1991).

9. J. Monk, Y. Yang, M. I. Mendelev, M. Asta, J. J. Hoyt and D. Y. Sun, "Determination of the crystal-melt interface kinetic coefficient from molecular dynamics simulations", Modelling Simul. Mater. Sci. Eng., 18, 015004(1-18) (2010). doi:10.1088/0965-0393/18/1/015004.

10. D.Y. Sun, M. Asta, and J. J. Hoyt, "Kinetic coefficient of Ni solid-liquid interfaces from molecular-dynamics simulations", Phys. Rev. B, 69, 024108 (2004). doi:10.1103/PhysRevB.69.024108

11. Y.F. Gao, Y.Yang, D.Y.Sun, M.Asta, J.J.Hoyt, "Molecular dynamics simulations of the crystalmelt interface mobility in HCP Mg and BCC Fe", J.Crystal Growth, 312 (21) 3238-3242 (2010). doi.org/10.1016/j.jcrysgro.2010.07.051

12. K.A. Jackson, Kinetic processes:crystal growth, diffusion, and phase transitions in materials, Weinheim: Wiley-VCH Verlag GmbH \& Co. KGaA, (2004). 
13. V.I. Mazhukin, "Kinetics and Dynamics of Phase Transformations in Metals Under Action of Ultra-Short High-Power Laser Pulses. Ch.8”, 219 -276, Laser Pulses - Theory, Technology, and Applications, I. Peshko (Ed.), InTech, Croatia, (2012). http://dx.doi.org/10.5772/50731

14. B. Chalmers, Principles of Solidification, N. Y. John Wiley \&Sons, (1964).

15. S.R. Coriell, D. Turnbull, "Relative roles of heat transport and interface rearrangement rates in the rapid growth of crystals in undercooled melts", Acta Metallurgica, 30 (12), 2135-2139 (1982).

16. M. Amini, B.B. Laird, "Kinetic coefficient for hard-sphere crystal growth from the melt", Phys. Rev. Lett., 97, 216102(1-4) (2006).

17. J.J. Hoyt, M. Asta, A. Karma, "Atomistic simulation methods for computing the kinetic coefficient in solid-liquid systems", Interface Science, 10 (2-3), 181-189 (2002).

18. M.I. Mendelev, M.J. Rahman, J.J. Hoyt, M. Asta, "Molecular-dynamics study of solid-liquid interface migration in fcc metals", Modelling Simul. Mater. Sci. Eng., 18, 074002(1-18) (2010). http://dx.doi.org/10.1088/0965-0393/18/7/074002

19. W. Kurz and D. J. Fisher, Fundamentals of Solidification, $3^{\text {rd }}$ ed., Trans Tech, Aedermannsdorf, Switzerland, (1992).

20. R.Trivedi, W.Kurz, "Solidification microstructures: A conceptual approach", Acta Metallurgica et Materialia. 42 (1), 15-23 (1994). https://doi.org/10.1016/0956-7151(94)90044-2

21. P. Galenko, S. Sobolev. "Local nonequilibrium effect on undercooling in rapid solidification of alloys", Phys. Rev. E, 55 (1), 343 - 352 (1997).

22. D. M.Herlach, "Solidification from undercooled melts", Materials Science and Engineering: A, 226-228, 348-356 (1997). https://doi.org/10.1016/S0921-5093(96)10644-4

23. Y. Ashkenazy, R. S. Averback, "Atomic mechanisms controlling crystallization behaviour in metals at deep undercoolings", Europhysics Letters (EPL), 79 (2), 26005(1-6) (2007). doi: $10.1209 / 0295-5075 / 79 / 26005$

24. V.I. Mazhukin, "Nanosecond laser ablation: mathematical models, computational algorithms, modeling Chapter 2", 31 -55, Laser Ablation - From Fundamentals to Applications, Tatiana Itina (Ed.), InTech, Croatia, (2017).

25. C. A. MacDonald, A. M. Malvezzi, \& F. Spaepen, "Picosecond time-resolved measurements of crystallization in noble metals", JAP, 65 (1), 129-136, (1989). doi:10.1063/1.342586

26. M. B. Agranat, S. I. Ashitkov, V. E. Fortov, A. V. Kirillin, A. V. Kostanovskii, S. I. Anisimov, \& P. S. Kondratenko, "Use of optical anisotropy for study of ultrafast phase transformations at solid surfaces", Appl. Phys. A: Materials Science \& Processing, 69(6), 637-640, (1999). doi:10.1007/s003390051045

27. W. L. Chan, R. S. Averback, D. G. Cahill, Y. Ashkenazy, "Solidification velocities in deeply undercooked silver", Phys. Rev. Lett., 102(9), 095701(1-4) (2009). https://doi.org/10.1103/PhysRevLett.102.095701

28. V.I. Mazhukin, A.V. Shapranov, M.M. Demin, N.A. Kozlovskaya, "Temperature dependence of the kinetics rate of the melting and crystallization of aluminum", Bulletin of the Lebedev Physics Institute, 43 (9), 283-286 (2016).

29. V. I. Mazhukin, A. V. Shapranov, V. E. Perezhigin, O. N. Koroleva, A. V. Mazhukin, "Kinetic melting and crystallization stages of strongly superheated and supercooled metals", Mathematical Models and Computer Simulations, 9 (4), 448-456 (2017).

30. H.A. Wilson, "On the velocity of solidification and viscosity of supercooled liquids", Philos. Mag., 50, 238-250 (1900).

31. J.I. Frenkel, "Note on the relation between the speed of crystallization and viscosity", Phys. Z. Sowjet Union, 1, 498 - 499 (1932).

32. J. Frenkel, Kinetic Theory of Solids, Oxford University Press, N. Y., (1946).

33. J.Q. Broughton, G.H. Gilmer, K.A. Jackson, "Crystallization Rates of a Lennard-Jones Liquid", Phys. Rev. Let., 49, 1496 -1500 (1982). 
34. L.V. Mikheev, A. A. Chernov, "Mobility of a diffuse simple crystal-melt interface", J. Crystal Growth, 112 (2-3), 591-596 (1991). doi:10.1016/0022-0248(91)90340-b

35. Y. Ashkenazy, R.S. Averback, "Kinetic stages in the crystallization of deeply undercooled bodycentered-cubic and face-centered-cubic metals", Acta Materialia, 58, 524-530 (2010).

36. D. Turnbull, "On the relation between crystallization rate and liquid structure", J. Phys. Chem., 62 (4), $609-613$ (1962).

37. J.E. Jones, "On the determination of molecular fields. I. From the Variation of the Viscosity of a Gas with Temperature", Proc. Royal Society of London. Series A, 106 (738), 441-462 (1924).

38. V.V. Zhakhovskii, N.A. Inogamov, Yu.V. Petrov, S.I. Ashitkov, K. Nishihara, "Molecular dynamics simulation of femtosecond ablation and spallation with different interatomic potentials", Appl. Surf. Sci., 255(24), 9592-9596 (2009).

39. S. M. Foiles, M. I. Baskes, M. S. Daw, "Embedded-Atom-Method Functions for the Fcc Metals $\mathrm{Cu}, \mathrm{Ag}, \mathrm{Au}, \mathrm{Ni}, \mathrm{Pd}, \mathrm{Pt}$ and their alloys", Phys. Rev. B, 33, 7983 -7991 (1986).

40. M.I. Mendelev, S. Han, D.J. Srolovitz, G.J. Ackland, D.Y. Sun, M. Asta, "Development of new interatomic potentials appropriate for crystalline and liquid iron", Philosophical Magazine, 83 (35), 3977-3994 (2003).

41. G.J. Ackland, M.I. Mendelev, D.J. Srolovitz, S. Han, A.V. Barashev, "Development of an interatomic potential for phosphorus impurities in $\alpha$-iron", J. Phys. Condens. Matter, 16, s2629 (1-14) (2004).

42. M.I. Mendelev, "Molecular dynamics simulation of solidification and devitrification in a onecomponent system", Modelling Simul. Mater. Sci. Eng., 20(4), 045014(1-17) (2012). https://doi.org/10.1088/0965-0393/20/4/045014

43. Q.S. Mei, K. Lu, "Melting and superheating of crystalline solids: From bulk to nanocrystals", Progress in Materials Science, 52 (8), 1175-1262 (2007).

44. V. I. Mazhukin, M. M. Demin, A. V. Shapranov, "High-speed laser ablation of metal with picoand subpicosecond pulses", Appl. Surf. Sci., $\underline{\mathbf{3 0 2}}, \quad 6-10$ (2014). https://doi.org/10.1016/j.apsusc.2014.01.111

45. Gang Sun, Jenny Xu \& Peter Harrowell, "The mechanism of the ultrafast crystal growth of pure metals from their melts", Nature Materials, 17, 881-886 (2018). doi: https://doi.org/10.1038/s41563-018-0174-6

46. Peter K. Galenko, Dmitri V. Alexandrov, "From atomistic interfaces to dendritic patterns", Medicine, Physics. Philosophical Transactions of the Royal Society A: Mathematical, Physical and Engineering Sciences, 376 (2113) (2018). doi:10.1098/rsta.2017.0210.

47. J. Schmelzer, T. Tropin, "Glass Transition, Crystallization of Glass-Forming Melts, and Entropy”, Entropy, 20(2), 103(1-32) (2018). doi:10.3390/e20020103

48. T. V. Tropin, J. W. Schmelzer \& V. L. Aksenov, "Modern aspects of the kinetic theory of glass transition", Physics-Uspekhi, 59(1), 42-66 (2016). doi:10.3367/ufne.0186.201601c.0047

49. V.I. Mazhukin, A.V. Shapranov, A.V. Mazhukin, O.N. Koroleva, "Mathematical formulation of a kinetic version of Stefan problem for heterogeneous melting/crystallization of metals", Mathematica Montisnigri, 36, 58-77 (2016).

50. M.D. Kluge, J.R. Ray, "Velocity versus temperature relation for solidification and melting of silicon: A molecular-dynamics study", Phys. Rev. B, 39 (3), 1738 -1746 (1989).

51. M.D. Kluge, J.R. Ray, "Pulsed laser melting of silicon: A molecular dynamics study", J.Chem. Phys., 87 (4), 2336 - 2339 (1987).

52. C.J. Tymczak, J.R. Ray, "Asymmetric Crystallization and Melting Kinetics in Sodium: A Molecular-Dynamics Study", Phys. Rev. Let., 64 (11), 1278 - 1281 (1990).

53. C.J. Tymczak, J.R. Ray, "Interface response function for a model of sodium. A molecular dynamics study", J. Chem. Phys., 92 (12), 7520 - 7530 (1990).

54. V.I. Mazhukin, A.V. Shapranov, A.V. Mazhukin, P.V. Breslavsky, "Atomistic modeling of the dynamics of the solid/liquid interface of Si melting and crystallization taking into account deeply 
superheated/supercooled states", Mathematica Montisnigri, 47, 87-99 (2020). doi: http://doi.org/10.20948/mathmontis-2020-47-8

55. B. Rethfeld, K. Sokolowski-Tinten, D. von der Linde, S.I. Anisimov, "Ultrafast thermal melting of laser-excited solids by homogeneous nucleation”, Phys. Rev. B, 65, 092103(1-4) (2002).

56. V.I. Mazhukin, A.V. Shapranov, V.E. Perezhigin, "Matematicheskoe modelirovanie teplofizicheskix svojstv, processov nagreva i plavleniya metallov metodom molekulyarnoj dinamiki”, Mathematica Montisnigri, 24, 47 - 66 (2012).

Received June 18, 2020 